\title{
Alerta Antecipado de Desastres para Deficiente Visual e Auditivo: Reportando Resultados de Uma Pesquisa de Campo
}

\author{
Jaziel Souza Lobo ${ }^{1,2}$, Vaninha Vieria ${ }^{1}$ \\ ${ }^{1}$ Universidade Federal da Bahia (UFBA) - Salvador - BA - Brasil \\ ${ }^{2}$ Instituto Federal de Sergipe - Campus São Cristóvão - Aracaju - SE - Brasil \\ jaziel.lobodifs.edu.br, vaninha.vieira@ufba.br
}

\begin{abstract}
Early Warning Systems were created to alert society to events that can lead to disaster situations. Most systems send the same alert to everyone, although there are vulnerable groups such as the blind and deaf who may have different needs. This article presents the results of an exploratory field survey with the objective of identifying the perception of members of the vulnerable group of blind and deaf people and professionals involved with deaf people about how these groups should be alerted. The results point to a direction on how accessible alerts can be sent to this group, as well as their difficulties and gaps in this area.
\end{abstract}

Resumo. Os sistemas de alerta antecipado foram criados para avisar antecipadamente a sociedade sobre eventos que possam gerar situações de desastre. Muitos destes sistemas enviam alertas iguais para todas as pessoas, apesar de existirem grupos vulneráveis como cegos e surdos que podem ter necessidades diferentes. Esse artigo apresenta os resultados de uma pesquisa de campo exploratório com o objetivo de identificar a percepção de membros do grupo vulnerável de cegos e surdos e de profissionais envolvidos com pessoas surdas sobre como deveriam ser alertados estes grupos. Os resultados apontam um direcionamento de como podem ser enviados alertas acessiveis a este grupo, bem como suas dificuldades e as lacunas nesta área.

\section{Introdução}

Ao longo dos anos, situações de emergência como terremotos, erupções vulcânicas, inundações, deslizamentos de terra ou incêndios têm afetado a vida das pessoas e gerado situações de crises, como as catástrofes naturais que ocorrem pelo mundo [Engelbrecht et al. 2011, He 2012]. A comunicação no gerenciamento de desastre é uma função crítica pois a disseminação de informações precisas e no momento certo para o público desempenha um papel importante para prevenir danos e vítimas [Skrbek 2011, Anhong et al. 2009]. Os Sistema de Alerta Antecipado, ou apenas EWS da sigla em inglês Early Warning Systems, são definidos como sistemas que possuem o conjunto de capacidades necessárias para gerar e disseminar avisos em tempo suficiente para que indivíduos, comunidades e organizações ameaçadas por um perigo se preparem e ajam adequadamente para reduzir a possibilidade de danos ou perdas [UNISDR 2009].

O principal motivo de alertar a população de forma antecipada em emergências é salvar vidas e prevenir ou reduzir os riscos de danos que podem ser causados pelo 
agravamento da situação. De acordo com a Secretaria Interinstitucional da Estratégia Internacional para a Redução de Desastres da ONU (UN/ISDR), para que um EWS ser eficaz, ele precisa ser centrado nas pessoas [United Nations 2006]. Apesar deste entendimento, [Meissen et al. 2014] relata que a maioria dos EWS utilizam a abordagem "uma mensagem para todos", disseminando de forma massiva uma mesma mensagem para todas as pessoas, sem fazer qualquer distinção de público-alvo, seja ele uma pessoa com deficiência, um idoso, uma pessoa do governo ou da imprensa.

Com o mesmo entendimento, [Sullivan and Häkkinen 2011] chamam a atenção para as populações vulneráveis como os deficientes que, em uma situação de desastre, estão sujeitas a um risco especial e, desta forma, torna-se fundamental que as suas necessidades sejam consideradas na concepção de sistemas de preparação e aviso de desastres. Corroborando com este pensamento, [Klafft and Reinhardt 2016] relatam que a Associação de Surdos da Baviera os abordou para destacar a importância de aplicativos de alerta de desastres adequados para seus membros.

Em [Malizia et al. 2010] foi analisado se os EWS consideravam princípios de acessibilidade. Neste estudo, os autores expõem que avaliaram nove sistemas e apenas um deles fornecia notificações acessíveis direcionadas para pessoas com deficiência auditiva através de uma tecnologia que, segundo o trabalho, era antiga e não padronizada. O que se percebe é que a personalização das mensagens de alerta está agora sendo aceita como uma forma de aumentar o alcance dos alertas. Esta percepção é ratificada no trabalho [Klafft and Reinhardt 2016], onde os gerentes de emergência concordam que diferentes grupos vulneráveis precisam de atenção e informações especiais, desde que todos (grupos vulneráveis ou não) recebam a mensagem padrão e que ao enviar mensagens adicionais para grupos vulneráveis, que elas sejam extensões da mensagem base e que todos possam entender o porquê receberam essas informações adicionais.

Diante deste cenário, esse artigo apresenta os resultados de uma pesquisa de campo realizada como primeiro passo de contato para conhecer pessoas cegas e pessoas surdas, e para identificar como poderiam ser enviados os alertas para estes grupos de pessoas. Este trabalho está inserido em um projeto maior, o projeto UAware, que visa desenvolver uma plataforma computacional inteligente de apoio à gestão de crises e emergências, com particular atenção ao problema da comunicação em crises decorrentes de desastres e emergências. O projeto apoia, principalmente, a comunicação entre o centro de comando e a comunidade afetada, imprensa, autoridades públicas, público em geral e primeiros socorristas.

Assim, esse artigo está estruturado da seguinte forma: a seção 2 apresenta o background teórico e os trabalhos relacionados. A Seção 3 apresenta a metodologia. Na Seção 4, os resultados são descritos. Por fim, na Seção 5 estão as conclusões deste estudo.

\section{Background Teórico e Trabalhos Relacionados}

Populações vulneráveis como as pessoas com deficiências estão sujeitas a um risco especial em um desastre e é fundamental que as suas necessidades sejam consideradas na concepção de sistemas de preparação e aviso de desastres [Sullivan and Häkkinen 2011]. Segundo dados da Organização Mundial da Saúde [WHO 2015], mais de um bilhão de pessoas, cerca de $15 \%$ da população mundial, possuem alguma forma de deficiência. No Brasil, este percentual é ainda maior, $23 \%$ da população, segundo o censo demográfico 
de 2010 do IBGE.

Por não serem um grupo homogêneo, as pessoas com deficiência possuem necessidades diferentes. Dessa forma, a necessidade de pessoas com deficiência auditiva é diferente das necessidades daqueles que são cegos. Para os cegos os smartphones disponibilizam leitores de tela como o TalkBack que convertem texto para voz através de uma tecnologia conhecida como TTS (Text-To-Speech). Estes aplicativos permitem que eventos lançados por outros aplicativos sejam capturados e lidos como, por exemplo, uma notificação de texto. Apesar da presença da tecnologia TTS, [Liu et al. 2015] relata que esta solução não é perfeita e que não são todos eventos lançados que são capturados pelos celulares.

Com relação aos deficientes auditivos, [Bisol et al. 2010] explica que pessoas surdas apresentam um baixo nível de letramento e dificuldades na aprendizagem da leitura e escrita de textos em português. Dessa forma, tem surgido pesquisas focadas em sistemas computacionais de tradução automática do português para a Língua Brasileira de Sinais (Libras), no entanto, segundo [Corrêa et al. 2018], ainda existe uma baixa produção sobre o tema. Assim sendo, no caso das pessoas surdas, é preciso que as mensagens sejam transmitidas em Libras.

Alguns estudos têm se preocupado com a questão da acessibilidade em sistemas móveis de forma geral, como por exemplo [Oliveira et al. 2020]. Neste estudo é investigada uma amostra de 24 sistemas governamentais digitais móveis do governo federal brasileiro, para examinar se os requisitos de acessibilidade foram incluídos no processo de desenvolvimento de tais sistemas. Dos 24 sistemas móveis avaliados, apenas sete levaram em consideração requisitos de acessibilidade.

Outros autores pesquisam acessibilidade para EWS, como, por exemplo, [Aedo et al. 2012, Malizia et al. 2009, Meissen and Voisard 2008, Mitchell et al. 2010, Rahman et al. 2012, Tyson et al. 2016, Vitalij et al. 2012], que propões soluções com algum suporte para pessoas com deficiência visual. Dentre o suporte oferecido nestes trabalhos, a maioria fazia uso de mensagens de áudio e o uso da tecnologia TTS. Já [Aedo et al. 2012, Malizia et al. 2009, Mitchell et al. 2010, Vitalij et al. 2012] apresentaram soluções para deficientes auditivos, fazendo uso de textos e vídeos em língua de sinais, além da utilização de padrões diferenciados de vibração do celular e o uso de imagens e vídeo para alertar este grupo de pessoas.

Em [Klafft and Reinhardt 2016] é apresentado um levantamento de requisitos de interação e informações adicionais para grupos vulneráveis que precisam ser levados em consideração por aplicativos de alerta de desastre para smartphones. De acordo com a investigação, para que pessoas cegas possam entender os alertas, elas necessitam de uma ferramenta que transforme o texto em fala e, se houver imagens, é preciso que se tenha uma descrição textual. Já para as pessoas surdas, os autores relatam que é difícil chamar a atenção dos surdos porque eles costumam ficar muito focados em um aspecto do ambiente (situação de "olhos ocupados"). Estes autores mencionam que muitas soluções utilizam lanternas, alertas de vibração em smartphones ou a conversão da tela do smartphone em uma espécie de lanterna, alternando constantemente entre branco brilhante e preto. Outra sugestão deste estudo é fornecer vídeos em linguagem de sinais que é a língua nativa das pessoas surdas. Apesar de avaliar os requisitos para grupos vulneráveis e avaliar 
alguns sistemas, [Klafft and Reinhardt 2016] concluem que não é possível se ter a clareza do quanto que os aplicativos de alerta atendam aos requisitos dos grupos da população vulnerável.

Foi possível notar nestes trabalhos que por mais que propunham soluções para grupos vulneráveis, alguns não utilizaram pessoas deficientes nos experimentos, e, quando utilizam, não apresentam os resultados destes grupos. Em [Mitchell et al. 2010] é explicitado um estudo com deficientes auditivos, mas neste caso foram utilizados vídeos na Linguagem de Sinais Americana (ASL). Por não ter sido encontrado pesquisas com público brasileiro que avaliem o envio de alerta antecipado sobre situações de desastres voltado para pessoas deficientes, tornou-se necessário realizar um estudo de campo para preencher essa lacuna. A próxima seção apresentará a metodologia utilizada na condução de uma pesquisa de campo para identificar através de entrevistas como alertar deficientes visuais e deficientes auditivos.

\section{Metodologia}

Esse trabalho é um estudo qualitativo exploratório, com entrevistas semiestruturadas para conhecer como alertar pessoas surdas e cegas. A pesquisa de campo que fundamenta este estudo foi realizada em dois momentos e contou com a participação voluntária de 7 deficientes visuais, 3 intérpretes de Libras e 1 deficiente auditivo.

Inicialmente, o estudo foi realizado com os deficientes visuais da Associação Baiana de Cegos - Salvador/BA. O contato com este grupo foi intermediado por um membro do grupo de pesquisa CEManTIKA. Sua proximidade com o presidente da associação permitiu que nos aproximássemos dos associados para a realização deste estudo. Antes das entrevistas, foi explicado para todos os integrantes o teor da pesquisa e lido o termo de consentimento livre esclarecido para que todos soubessem os objetivos deste trabalho. As entrevistas semiestruturadas foram realizadas individualmente e conduzidas por dois membros do grupo de pesquisa CEManTIKA, por cerca de quinze minutos de duração, tendo sido gravadas em áudio e posteriormente transcritas para análise.

No segundo momento, o estudo foi dedicado a avaliar o grupo de deficientes auditivos. Devido a proximidade com um intérprete da Língua Brasileira de Sinais (Libras), as primeiras entrevistas foram realizadas com intérpretes. As entrevistas foram realizadas com o apoio de um questionário impresso, gravadas em áudio e posteriormente transcritas para análise. Durante a entrevista, foram sugeridos nomes de pessoas surdas para serem entrevistadas. Das pessoas inicialmente citadas, apenas um conseguiu espaço na agenda para ser entrevistado, dificultando a amostra como também as análises dos resultados encontrado. A entrevista com o surdo foi realizada com o acompanhamento de um intérprete de Libras. Também foi utilizado um questionário como apoio e a entrevista foi gravada em áudio e transcrita.

\section{Resultados}

Os resultados das entrevistas serão apresentados separadamente para os grupos de deficientes visuais e deficientes auditivos, sendo organizados em três categorias. Na primeira, discute-se as perguntas iniciais da entrevista que abordavam dados gerais para caracterização do entrevistado. Na segunda, avaliam-se questões relativas ao envolvimento com às situações de desastres. Por fim, discutem-se aspectos relacionados como 
alertá-los (cegos e surdos) através do smartphone. Devido ao número limitado de participantes, os resultados devem ser considerados exploratórios e precisam ser confirmados em um estudo maior.

\subsection{Deficientes visuais}

Foram entrevistados 7 deficientes visuais, sendo 2 deles com baixa visão e 5 cegos. Nesta seção serão apresentados os dados de acordo com as categorias acima especificadas. Dos 7 entrevistados, dois tinham a deficiência desde o nascimento, uma pessoa cega e outra com baixa visão, os demais a deficiência foi adquirida ao longo da sua vida. Os dois entrevistados de baixa visão tinham 35 anos, enquanto as pessoas com cegueira tinham idade média de 53 anos, sendo a menor idade identificada de 38 anos e a maior de 67 anos. Considerando-se todos os entrevistados sem agrupamentos, a média de idade passa a ser de 48 anos.

Quando questionados sobre o envolvimento com alguma situação de desastres, $42,86 \%$ dos entrevistados informaram que nunca tinham qualquer envolvimento. Os outros $57,14 \%$ responderam que já se envolveram e citaram incêndio e alagamento como sendo os eventos ocorridos.

As perguntas da sequência tiveram o objetivo de identificar formas de alertar pessoas cegas sobre situações de desastre utilizando smartphones. Dessa forma, o primeiro item foi direcionado a saber como as pessoas que se envolveram em situações de desastres foram avisadas do evento, como eles achavam que poderiam ser alertadas e como o alerta poderia ser feito através do smartphone.

Questionando-se como haviam sido avisados sobre as situações de desastre que se envolveram, duas pessoas relataram que ouviram barulhos ou gritos que chamaram a sua atenção, uma pessoa informou que foi alertada por outra pessoa e outro entrevistado informou que não foi avisado, apenas sentiu a água no meio da cintura quando desceu as escadas de casa. Indagados como achavam que uma pessoa cega deveria ser avisada sobre os desastres, surgiram respostas como: através de aplicativos, através de dispositivos ou um alerta sonoro. As demais respostas remeteram a ajuda por outras pessoas ou não souberam responder.

Sobre possuir ou se já possuiu smartphones, 28,57\%, representando 2 entrevistados, informaram que possuíam, 28,57\% informaram que já tiveram, mas não possuem mais porque não se adaptaram, e os outros 42,86\% (3 entrevistados) responderam que não possuíam smartphone.

\subsection{Deficientes auditivos}

Foram entrevistadas 4 pessoas, das quais uma era surda e três eram intérpretes de libras. Traçando um perfil dos intérpretes, um atuava apenas como intérprete e os outros dois como professores de Libras, sendo um no nível superior e o outro no nível médio. Todos tinham experiência com Libras a mais de 15 anos, inclusive um dos professores já lecionava a mais de 28 anos. Apenas um dos intérpretes tinha se envolvido em uma situação de desastre que foi uma explosão em um gasoduto, que afetou o bairro onde morava, mas o entrevistado relatou que não recebeu qualquer aviso ou alerta antes ou depois do incidente. $\mathrm{O}$ entrevistado surdo possuía 26 anos quando foi entrevistado. Ele é surdo desde 
o nascimento e sua primeira língua aprendida foi Libras. O entrevistado também relatou em sua entrevista que nunca se envolveu em uma situação de desastres.

Com relação ao conhecimento sobre sistemas de alerta antecipado, todos os participantes responderam que já tinham ouvido falar ou conheciam tais sistemas, embora todos também tenham relatado nunca receberem nenhum alerta até aquele momento. Para este grupo de entrevistado, foi questionado se eles moravam em área considerada de risco e todos informaram que não.

Neste momento as perguntas foram direcionadas para como alertar pessoas surdas sobre situações de desastre através do smartphone. Os resultados mostraram um alinhamento das respostas dos intérpretes com o surdo entrevistado. Por unanimidade, todos entrevistados responderam que o alerta deveria ser dado em Libras. Outras sugestões como imagens ou desenhos como os utilizados por placas de trânsito e animações foram citadas pelo entrevistado surdo. As Tabelas 1 e 2 resumem como deveriam ser os alertas e os recursos do smartphone que deveriam ser utilizados para chamar a atenção dos surdos.

Tabela 1. Formas de alerta para pessoas surdas

\begin{tabular}{|l|c|r|r|}
\hline \multicolumn{1}{|c|}{ Como alertar? } & $\begin{array}{c}\text { Entrevistado } \\
\text { Surdo }\end{array}$ & Intérpretes & TOTAL \\
\hline Em Libras & $25 \%(1)$ & $75 \%(3)$ & $100 \%(4)$ \\
Desenhos ou animações & $50 \%(1)$ & $50 \%(1)$ & $50 \%(2)$ \\
\hline
\end{tabular}

Tabela 2. Formas de chamar a atenção de pessoas surdas para o smartphone

\begin{tabular}{|l|l|l|l|}
\hline Como chamar a atenção? & Entrevistado Surdo & Intérpretes & TOTAL \\
\hline Sinal Luminoso & $25,00 \%(1)$ & $75,00 \%(3)$ & $100 \%(4)$ \\
Vibração & $33,33 \%(1)$ & $66,67 \%(2)$ & $100 \%(3)$ \\
Luzes piscando & & $100,00 \%(1)$ & $100 \%(1)$ \\
Cores variadas & & $100,00 \%(2)$ & $100 \%(2)$ \\
\hline
\end{tabular}

\section{Conclusões}

Esse é um estudo qualitativo, exploratório, com uma amostra pequena, que busca fontes distintas na tentativa de sugerir como alertar pessoas cegas ou surdas para situações de desastres através do smartphone. Até onde se pesquisou, são poucos estudos sobre sistemas de alerta antecipado que discutem a adaptação do envio dos alertas para estes grupos vulneráveis. Esse foi o primeiro passo em direção a um sistema de alerta antecipado de desastres acessível. A personalização das mensagens para os deficientes visuais e deficientes auditivos torna-se importante pois pode significar salvar vidas.

A revisão da literatura indicava que os surdos apresentam dificuldades na leitura de textos em português. Durante as entrevistas com os intérpretes também foi relatado esse fato, sendo este o motivo de todos indicarem que os alertas deveriam ser em Libras. $\mathrm{Na}$ entrevista da pessoa surda, foi possível constatar tal situação, quando foi apresentado ao entrevistado um texto sobre um alerta e posteriormente foi solicitado que ele dissesse o que ele entendeu do texto. Também ficou claro na entrevista que não basta só traduzir o texto para Libras, é preciso adaptar o texto para que ele seja mais claro e tenha um 
vocabulário que consiga ser de fácil entendimento. Dessa forma, é preciso que seja realizado um estudo aprofundado sobre qual o vocabulário adequado para ser utilizado no envio de mensagens de alerta para pessoas surdas. Para a realização de entrevistas com pessoas surdas também foi respondido por todos os intérpretes que é possível realizá-las em grupo, desde que se tenha um intérprete no processo.

Com esse estudo percebe-se que um sistema de alerta antecipado deverá incluir o envio de alertas personalizados para os deficientes visuais e deficientes auditivos. No caso dos deficientes auditivos a mensagem deve ser enviada em Libras. Uma vez que Libras é uma linguagem brasileira, os dados aqui sinalizados podem divergir em outros países.

No caso dos deficientes visuais, é preciso que os sistemas de alerta antecipado contemplem de formas diferentes pessoas com baixa visão e pessoa cegas. Apesar da boa compreensão, também é preciso avaliar a utilização de termos técnicos nas mensagens de alerta direcionadas a este público. As entrevistas apontam para o envio de mensagens de áudio e a emissão de um beep antes da reprodução da mensagem.

\section{Referências}

Aedo, I., Yu, S., Díaz, P., Acuña, P., and Onorati, T. (2012). Personalized alert notifications and evacuation routes in indoor environments. Sensors, 12(6):7804-7827.

Anhong, L., Xiang, L., Wenjuan, F., Ning, A., Jian, Z., Lian, L., and Yongzhong, S. (2009). Blue arrow: A web-based spatially-enabled decision support system for emergency evacuation planning. 2009 Int. Conf. Bus. Intell. Financ. Eng. BIFE 2009, pages $575-578$.

Bisol, C. A., Valentini, C. B., Simioni, J. L., and Zanchin, J. (2010). Deaf Students in higher education: Reflections on inclusion. Cadernos de Pesquisa, 40:147 - 172.

Corrêa, Y., Giotti, J., Cruz, C. R., and Ribeiro, V. G. (2018). Produção científica brasileira sobre tradução automática português brasileiro-libras: uma revisão sistemática de literatura. Revista virtual de estudos da linguagem. Novo Hamburgo, RS. p. 179-203.

Engelbrecht, A., Borges, M. R. S., and Vivacqua, A. S. (2011). Digital tabletops for situational awareness in emergency situations. In Proceedings of the 2011 15th International Conference on Computer Supported Cooperative Work in Design (CSCWD), pages 669-676.

He, X. (2012). Study on emergency supply chain system planning under uncertainty. In 2012 International Conference on Information Management, Innovation Management and Industrial Engineering, volume 3, pages 432-435.

Klafft, M. and Reinhardt, N. (2016). Information and interaction needs of vulnerable groups with regard to disaster alert apps. In Weyers, B. and Dittmar, A., editors, Mensch und Computer 2016 - Workshopband, Aachen. Gesellschaft für Informatik e.V.

Liu, K. C., Wu, C. H., Tseng, S. Y., and Tsai, Y. T. (2015). Voice helper: A mobile assistive system for visually impaired persons. Proc. - 15th IEEE Int. Conf. Comput. Inf. Technol. CIT 2015, 14th IEEE Int. Conf. Ubiquitous Comput. Commun. IUCC 2015, 13th IEEE Int. Conf. Dependable, Auton. Se, pages 1400-1405. 
Malizia, A., Acuna, P., Onorati, T., Diaz, P., and Aedo, I. (2009). CAP-ONES: an emergency notification system for all. International Journal of Emergency Management, 6(3/4):302.

Malizia, A., Onorati, T., Diaz, P., Aedo, I., and Astorga-Paliza, F. (2010). Sema4a: An ontology for emergency notification systems accessibility. Expert Systems with Applications, 37(4):3380 - 3391.

Meissen, U., Hardt, M., and Voisard, A. (2014). Towards a general system design for community-centered crisis and emergency warning systems. ISCRAM 2014 Conference Proceedings - 11th International Conference on Information Systems for Crisis Response and Management, pages 155-159. cited By 0.

Meissen, U. and Voisard, A. (2008). Increasing the Effectiveness of Early Warning via Context-aware Alerting. Proceedings of the 5th International Conference on Information Systems for Crisis Response and Management ISCRAM2008, (May):431-440.

Mitchell, H., Johnson, J., and LaForce, S. (2010). The human side of regulation: Emergency alerts. In Proceedings of the 8th International Conference on Advances in Mobile Computing and Multimedia, MoMM '10, pages 180-187, New York, NY, USA. ACM.

Oliveira, A. C., da Silva, L. F., Eler, M. M., and Freire, A. P. (2020). Do brazilian federal agencies specify accessibility requirements for the development of their mobile apps? In XVI Brazilian Symposium on Information Systems, SBSI'20, New York, NY, USA. Association for Computing Machinery.

Rahman, K. M., Alam, T., and Chowdhury, M. (2012). Location based early disaster warning and evacuation system on mobile phones using openstreetmap. In 2012 IEEE Conference on Open Systems, pages 1-6.

Skrbek, J. (2011). RADIO-HELP AS A SMART EARLY WARNING AND NOTIFICATION SYSTEM.

Sullivan, H. T. and Häkkinen, M. T. (2011). Preparedness and warning systems for populations with special needs: Ensuring everyone gets the message (and knows what to do). Geotechnical and Geological Engineering, 29(3):225-236.

Tyson, G., Bigham, J., Bodanese, E., Akhtar, N., Biswas, P., Langdon, P., Mimrot, V., Mukhopadhyay, P., and Ribeiro, J. V. (2016). Designing an adaptive emergency warning system for heterogeneous environments. In 2016 IEEE 27th Annual International Symposium on Personal, Indoor, and Mobile Radio Communications (PIMRC), pages $1-6$.

UNISDR (2009). UNISDR Terminoology on Disaster Risk Reduction. Geneva,Switzerland.

United Nations (2006). Global Survey of Early Warning Systems. Technical report.

Vitalij, F., Robnik, A., and Alexey, T. (2012). "safe city-an open and reliable solution for a safe and smart city. Elektrotehniski Vestnik, 79(5):262-267.

WHO (2015). WHO global disability action plan 2014-2021. Better health for all people with disability. 